\title{
The Italian contribution to the construction of the linac for the European spallation source
}

\author{
S. Gammino ${ }^{1}$ (D) A. Fabris ${ }^{2} \cdot$ M. Lindroos $^{3}$
}

Received: 11 February 2021 / Accepted: 25 April 2021 / Published online: 25 May 2021

(c) The Author(s) 2021, corrected publication 2021

\begin{abstract}
The European spallation source (ESS) uses a linear accelerator (linac) to deliver the high intensity proton beam to the target station for producing intense beams of neutrons. At the exit of the linac, the proton beam will have $2 \mathrm{GeV}$ energy and $62.5 \mathrm{~mA}$ current. The construction of an accelerator with the contribution of different laboratories is not a new concept but so far the laboratories were controlled by the same government (e.g. in USA and Japan) or they delivered components for an intergovernmental institution like CERN. The European Spallation Source is a research facility that gathers 40 active in-kind (IK) contributors from 13 States, even outside the European Union, so its construction is not only a technical and scientific challenge, but also an economic, political and social experiment. The case of the Italian contribution is interesting because of the structure of Italian industrial ecosystem, mostly based on small and medium-sized enterprises (SME), which may be unsuitable for the case of a research infrastructure which construction requires a high level of R\&D investments. Conversely, the well-known flexibility of SME to adapt to the requirements have balanced the weakness and the results are satisfactory. Following the overview of the Linac design, the paper will focus on the key issues of the Italian contribution, the state of the project ( $73 \%$ completion up to now) along with the point of view of the ESS management and the lesson learnt; the major outcomes for the economy and society will complete the discussion.
\end{abstract}

Keywords Protons · Neutrons - Spallation source $\cdot$ Linac $\cdot$ Research infrastructures · Accelerators technology $\cdot$ Socio-economic impact

$凶$ S. Gammino

gammino@lns.infn.it

1 Istituto Nazionale di Fisica Nucleare - Laboratori Nazionali del Sud, 95123 Catania, Italy

2 Elettra Sincrotrone Trieste- AREA Science Park, 34149 Basovizza, Italy

3 European Spallation Source ERIC, 22592 Lund, Sweden 


\section{Description of the European spallation source}

Many nuclear research reactors in Europe have come (or are coming) to the end of their technical life span. It is increasingly harder to win acceptance for building new research reactors at universities that often are located in or close to big cities. The scientists who primarily use neutrons for research are looking for new neutron sources to replace the research reactors. When in full operation, the European Spallation Source will have the capacity to support the experimental activities of about 3000 scientists. Not all these people will come to Lund in Sweden for every experiment, some might even choose to follow the experiment remotely and get their data over the grid from the ESS computer center in Copenhagen. However, the neutrons, the instruments in which measurements are done and the sample preparation and handling labs will all be at ESS. The hope is that ESS will become the preferred meeting place for neutron scientists for conferences, workshops and other meetings.

Neutrons at ESS are generated through spallation [1-3]. The "driver" of this process is an intense proton beam, which at ESS is generated with a proton linear accelerator. The accelerated protons hit a rotating tungsten target in which the spallation process takes place. The neutrons generated fly out of the target and are captured "above" in a new type of flat moderator in which they are "cooled". The cold and very bright neutron beam shines from the moderator surface towards the instruments where the material studies are done. A more traditional high-flux moderator is also being designed for a position "below" the target $[4,5]$. The instruments come in many different types to enable all kind of studies for example structural studies, studies of material dynamics, life sciences, soft matter and neutron imaging.

To become the world's most powerful neutron source, ESS will have a $5 \mathrm{MW}$ proton beam when fully operational. The pulses will be almost $3 \mathrm{~ms}$ long with a repetition rate of $14 \mathrm{~Hz}$. This is a new regime for spallation sources which usually have been operating with very short proton pulses of a few microsecond [6, 7]. The longer pulse will permit the moderators to fully saturate and with neutron choppers and long neutron flight times the neutron beam can be altered to fit different kinds of experiments [8].

To ultimately generate a $5 \mathrm{MW}$ proton beam in $3 \mathrm{~ms}$ long pulses at $14 \mathrm{~Hz}$ with a linear accelerator is very challenging and has not been done before. To keep costs down the superconducting cavities are required to operate at very high gradients, which are state of the art for this technology [9, 10].

Primary proton beam losses must be kept to a minimum to avoid activation of the accelerator and the tunnel; the beam must be distributed over a larger part of the target surface to not melt the target and the target itself must always rotate to not be damaged by the beam. Even the moderator will operate under challenging conditions and so will many experiments, which have to handle a lot of data and many samples. To assure that the proton beam is well focused and that losses are kept to a minimum, advanced beam instrumentation is used to observe the beam and correct for any possible miss steering or malfunctioning. A Machine Protection System [11] is always operational to dump the beam safely within $3 \mu$ s in case of any possible malfunctioning. An independent and safety certified access system assures that nobody can enter any area in which harmful levels of radiation are generated. While safety always comes first, a very high 

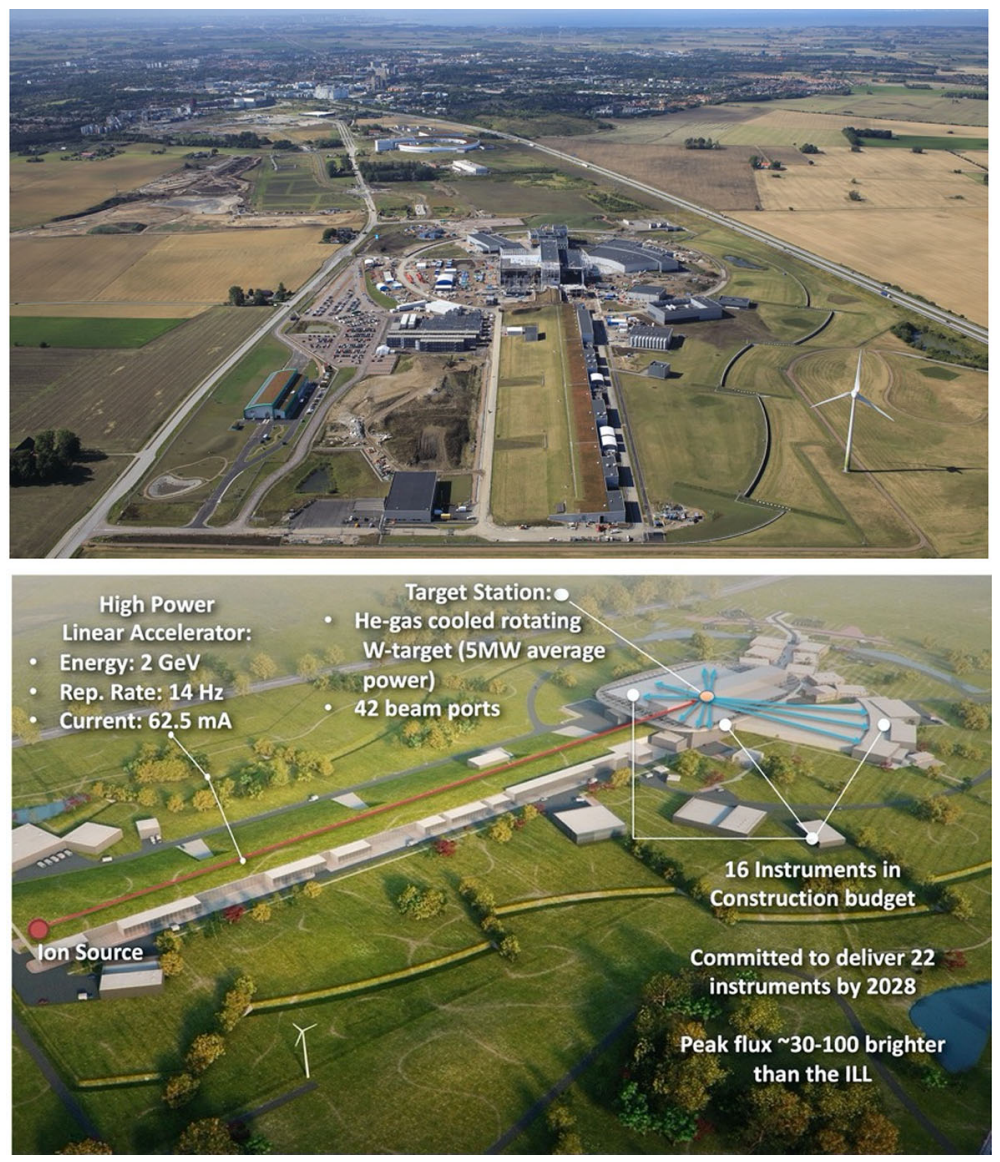

Fig. 1 View over the European Spallation Source (ESS) building: ESS will be the world's most powerful source of neutrons. A genuine pan-European facility, it will serve a community of 3,000 researchers across many areas of science and technology

availability of neutrons for users is a prime concern for ESS and every effort is being made to design and build a highly reliable facility.

To build ESS at a green field site in north Europe (Fig. 1) requires European collaboration, over 40 institutes and universities contribute in-kind. The in-kind model means that the ESS member states pay their own research institutes and universities to contribute to the construction of ESS in Lund with know-how, infrastructures for prototyping and testing, manufacturing of components, services and staff. Without the in-kind contributions ESS would have had to first recruit and train staff before to start to design and build ESS. This would have required much more time and would probably have been more costly than doing it through this in-kind collaboration model.

Italy is one of the founding members of the European Spallation Source ERIC (European Research Infrastructure Consortium), contributing with roughly $6 \%$ to the construction budget. The Italian government has designated INFN, CNR, Elettra Sin- 
crotrone Trieste (Elettra) as the three national Institutions in charge of realizing the Italian in-kind contribution to the project. In this framework INFN has been nominated as Representing Entity of Italy in the ERIC.

The accelerator receives in-kind contributions from some 22 partners from all over Europe for more than $50 \%$ of the budgeted cost. The partners for the ESS accelerator are INFN and Elettra in Italy, CEA and CNRS in France, STFC in UK, ISA in Denmark, Uppsala University and Lund University in Sweden, Atomki in Hungary, ESS-Bilbao in Spain and IFJPAN, Wroclaw University of Science and Technology, National Center of Nuclear Research, Lodz University and Warzaw University of Technology in Poland. They all contributed or are in some cases still contributing with unique know-how essential to make ESS possible.

The Normal Conducting (NC) section of the ESS accelerator consists of a Proton Source (PS), Low Energy Beam Transport (LEBT), Radio Frequency Quadrupole (RFQ), Medium Energy Beam Transport (MEBT), and Drift Tube Linac (DTL). The design, construction and testing of those structures is done by European partner labs as an in-kind contribution to the ESS project; in particular the Proton Source and the LEBT have been designed and built at INFN as a part of the Italian in-kind contribution, the RFQ has been provided by CEA, France, the MEBT by ESS-Bilbao, Spain, and the DTL is also an Italian contribution by INFN. The supervision of the normal conducting Linac has been assigned to Italy.

The Superconducting section of the Linac is also provided as in-kind contribution, involving different Institutions in Europe: the Spoke Cavities are provided by IN2P3, the Medium Beta Cavities (MB) by INFN, the High Beta Cavities (HB) by STFC, the Cryomodules (CM) by CEA and CNRS. Magnets, Power converters, Radio Frequency (RF) systems, diagnostics are to be provided by different Research Institutions and Elettra Sincrotrone Trieste is a major contributor.

The Italian in-kind contribution is completed by contributions of Consiglio Nazionale delle Ricerche (CNR) to the instruments for Neutron Science. More information about the ESS may be found in [1-3].

\section{The high power proton accelerator}

The accelerator design took advantage of the large amount of R\&D on High Power Proton Accelerators provided by some Research Institutions, in particular INFN and CEA, that worked in the Nineties to the design of waste transmutation facilities (namely TRASCO [12] and IPHI [13]). Additionally a large experience in the design and construction of superconducting cavities was obtained by INFN in the past decade with different projects, and the same can be said for Radiofrequency (RF) systems, magnets, power converters and diagnostics at Elettra Sincrotrone Trieste [14], that besides having built and upgraded its own accelerators based research facilities has provided relevant contributions to the construction of other facilities [see for example $15,16]$.

The high-power LINAC of the European Spallation Source (ESS) is designed to accelerate $62.5 \mathrm{~mA}$ of protons up to $2 \mathrm{GeV}$ in a sequence of normal conducting and superconducting accelerating structures. In Fig. 2 the sequence of sections is shown. 


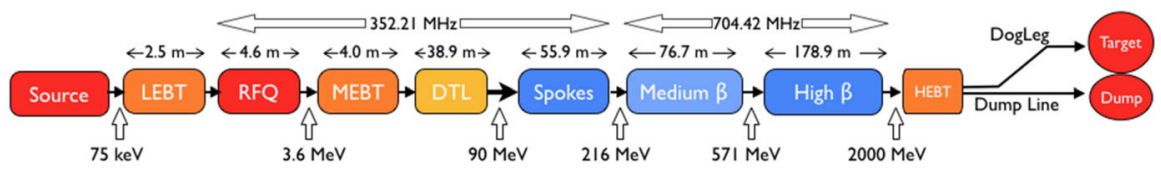

Fig. 2 Layout of the ESS linac

The accelerator is a $5 \mathrm{MW}$ proton linac delivering beams of energies up to $2.0 \mathrm{GeV}$ to the target in long pulses of $2.86 \mathrm{~ms}$ with a repetition rate of $14 \mathrm{~Hz}$ corresponding to a duty cycle of $4 \%$. Hands-on maintenance and machine protection set limits on beam losses, out of the RFQ, of $1 \mathrm{~W} / \mathrm{m}$ and $0.1 \mathrm{~W} / \mathrm{m}$ respectively $[17,18]$, and they are a major concern as it is for every high power linac. Therefore, it was crucial to design a linac which is expected to minimize the beam halo [19].

The proton linac is also designed for high reliability and the technical choices for each section were driven by this requirement, that is particularly stringent for superconducting cavities.

A normal conducting radio frequency quadrupole (RFQ) followed by a DTL section accelerate the protons supplied by a microwave discharge ion source up to a kinetic energy of $90 \mathrm{MeV}$. Then the acceleration is achieved by means of superconducting accelerating structures of three different types: 'Spoke' cavities are used up to $216 \mathrm{MeV}$, followed by medium- $\beta$ 6-cell elliptical cavities up to $571 \mathrm{MeV}$ and finally high- $\beta$ 5-cell cavities up to $2 \mathrm{GeV}$. A peak RF power of $150 \mathrm{MW}$ is necessary to get the nominal accelerating gradient in the Linac. A cryogenic facility provides the cooling power required to keep the superconducting cavities at a temperature of $2 \mathrm{~K}$. Water cooling is used for the normal conducting structures and all high power equipment (klystrons, RF loads, modulators, etc.).

The attention to sustainability and operational cost is coherent with the will to minimize the environmental footprint; it sets constraints on power requirements, power standardization and reliability, efficiency optimization. The RF systems represent an important contribution to the operational costs but is far from being the only one.

The requirements of high power and high reliability imply a central role of the computer control and of the machine protection system (MPS), and it puts a number of constraints over the design of each part of the accelerator. Even the diagnostics plays a major role and redundancy was considered.

The software environment of the control system is based on EPICS. It covers the whole facility including the slow controls for instruments and conventional facilities (CF). The machine protection system (MPS) takes care of avoiding damage to the equipment. The personnel safety system (PSS) handles the monitoring and access equipment required to ensure safety on site.

The Machine Protection System is designed to protect the accelerator from unintended beam loss, which could cause damage to the equipment [11]. It takes input from beam loss monitors and combined with beam veto signals from all equipment it can within microseconds abort the beam and dump it safely at low energy. The Personal Safety System (PSS) protects the staff from accidentally entering any area with dangerous levels of ionizing radiation. 

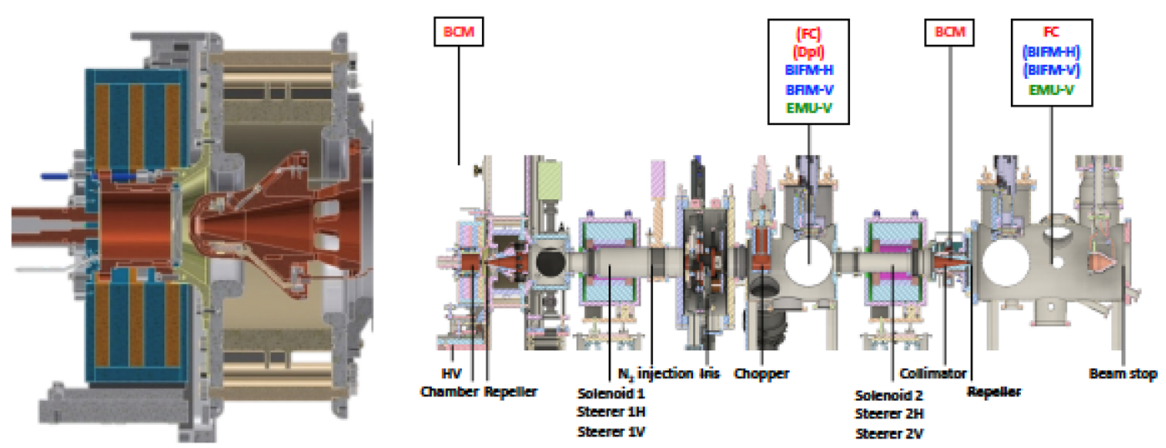

Fig. 3 Left: Scheme of PS-ESS chamber and extraction system. Right: Schematic of the LEBT

\subsection{Ion source and LEBT}

The high intensity beam of protons is produced by PS-ESS (Proton Source for ESS, Fig. 3 left), a Microwave Discharge Ion Source (MDIS) extracting the beam from a plasma generated by a $2.45 \mathrm{GHz}$ magnetron. The $3 \mathrm{~ms}-80 \mathrm{~mA}$ proton beam pulse generated by the PS-ESS with an energy of $75 \mathrm{keV}$ is transferred to the RFQ by means of the low energy beam transport line (LEBT, Fig. 3 right), which is composed of two magnetic solenoids that match the beam to the downstream RFQ, a chopper system that removes low quality head and tail of the beam, an iris that is used to decrease the beam current without affecting the beam quality, and a set of beam diagnostics that measures the beam before the injection in the linac.

During the factory tests at INFN-LNS, a detailed beam parameters characterization, upstream the matching point and at the matching point position, was carried out and it is used now for the Normal conducting Linac commissioning, to get rid of the unavailability of diagnostics at the RFQ matching point [20].

MDIS has been chosen because it has a reliability close to $100 \%$ and a long mean time between failures, MTBF, as demonstrated in similar ion sources [20, 21]. The PSESS and LEBT were manufactured and tested with beam at INFN-LNS then delivered to ESS at the end of 2017 (a second PS-ESS has been delivered in February 2020). Beam commissioning of these sections at the ESS site started in September 2018. In $[22,23]$ an overview on this first beam commissioning period at ESS, with the results of the source characterization and testing on LEBT functionalities, has been provided.

\subsection{RFQ}

The four-vane RFQ, as the first rf accelerating structure in the ESS linac, accelerates, focuses, and bunches the continuous $75 \mathrm{keV}$ beam to $3.62 \mathrm{MeV}$ within $4.6 \mathrm{~m}$ [24]. The beam current at the exit of the RFQ under nominal operation modes should be $62.5 \mathrm{~mA}$; taking into account the nominal transmission of $98 \%$, the input beam should be at least $64 \mathrm{~mA}$. The rf frequency of the RFQ is $352.21 \mathrm{MHz}$. The peak electric fields on the vane surface have been limited to 1.8 times the Kilpatrick value. 


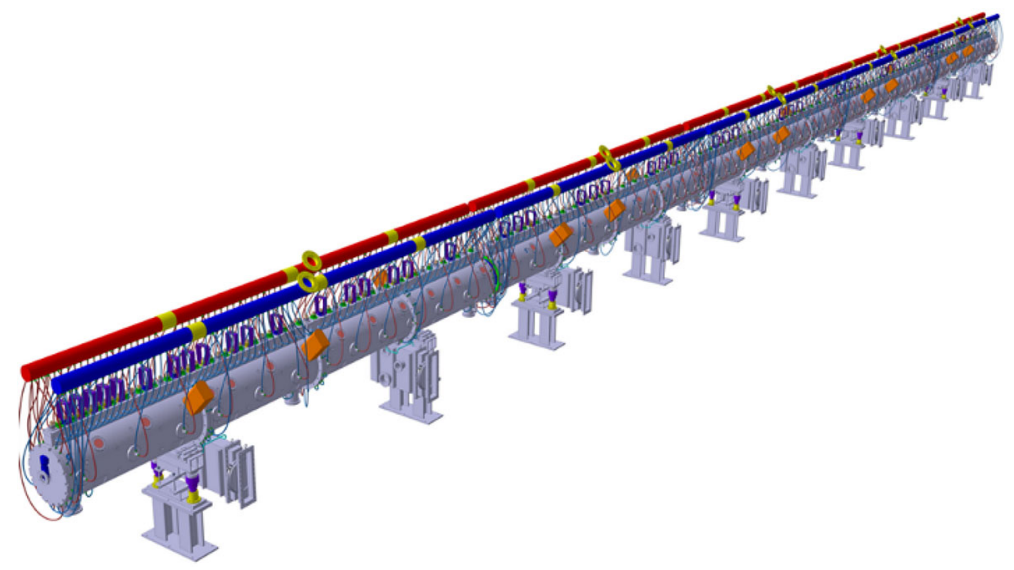

Fig. 4 Layout of the Drift Tube Linac

\subsection{MEBT}

A medium energy beam transport between the RFQ and the DTL [25] transports and matches the beam out of the former structure to the latter one, provides means to collimate the beam, measures the beam's transverse and longitudinal profile and chops off the remaining low-quality bunches which have not been cleaned out by the LEBT chopper. The MEBT is composed of 11 quadrupoles, three buncher cavities, a chopper with its dump system, 3 sets of 4 independent collimating plates and beam diagnostics.

\subsection{DTL}

The drift tube linac brings the beam energy to $89.6 \mathrm{MeV}$ in five tanks (Fig. 4). Each tank is fed by a $2.8 \mathrm{MW}$ klystron, having left a $30 \%$ margin for LLRF, tuning and waveguide losses; $2.2 \mathrm{MW}$ of power is delivered to the cavity via two RF windows, almost $50 \%$ of which is transferred to the beam. With respect to the original design, a higher input energy to the DTL was considered, which resulted in longer input cells and several positive consequences [26]; longer cells could house bigger quadrupoles reducing their magnetic gradient for the same integrated gradient, have longer gaps reducing the field at the flat of the drift tube and enhance the effective shunt impedance. The transverse focusing is still provided by permanent magnet quadrupoles (PMQs) that are housed in every other drift tube. Three corrector dipoles per plane per tank are correcting the beam centroid. The constraints present in a DTL required an optimization process on where to put these corrector dipoles. 


\subsection{Spoke section}

The DTL is the last normal conducting structure, and right after it comes the low energy differential pumping section, LEDP, that is used to create the required vacuum quality at the transition to superconducting cavities.

The spoke cavity linac is the first superconducting section of the accelerator complex. The 26 spoke cavities [27] will be used to accelerate the beam from 89.6 to $216 \mathrm{MeV}$. One of the reasons for choosing spoke cavities instead of the conventional normal-conducting structures in this energy range is their relatively large transverse aperture and tuneability for different phase and energy beams. These $352.21 \mathrm{MHz}$ double-spoke cavities with an optimum $\beta$ of 0.50 are housed in pairs in 13 cryomodules, and are separated by Linac warm units (LWU). As for the magnets in each LWU there are two quadrupoles and one dual plane corrector; a central slot is allocated to beam diagnostics.

\subsection{Elliptical sections}

The RF frequency doubles to $704.42 \mathrm{MHz}$ at the beginning of the next structure, the medium- $\beta$ elliptical cavities (Fig. 5). There are two families of elliptical cavities, accelerating the beam from the spoke output energy to $571 \mathrm{MeV}$ by using 36 medium- $\beta$ cavities and further to $2.0 \mathrm{GeV}$ by 84 high- $\beta$ cavities. In both sections four cavities are housed in cryomodules of identical length. Having different geometric $\beta$ s of 0.67 and 0.86 respectively, the medium- $\beta$ cavities are given an extra cell (6-cell) with respect to high- $\beta$ cavities (5-cell) to have almost the same length. There are identical elliptical warm units, elliptical LWUs, before each cryomodule. These LWUs have the same functionality as medium LWUs, with bigger apertures, and longer quadrupoles. To have the same flexibility at the spoke to medium- $\beta$ transition the period lengths in elliptical section is chosen to be exactly twice that of the spoke section [28, 29].

\subsection{HEBT}

The same periodicity, in transverse plane, is maintained for 15 periods after the high- $\beta$ section in the high energy beam transport, HEBT, for contingency HEBT purposes [1]. After this contingency area, there is one more LWUs which is followed by a vertical dipole with a bending angle of $4^{\circ}$ that also works as a switch magnet between the beam dump and the target. The beam going to the target is bent back to horizontal using a second vertical dipole after 6 periods of longer doublet focused sections that are adjusted to create an achromat dogleg. This beam is transported to the target using a set of quadrupoles and 8 raster magnets that paint the target surface in horizontal and vertical directions at different frequencies. To reduce the beam center movement on target due to energy jitter the phase advance between the second dipole and the target surface is set to be a multiple $180^{\circ}$. A fixed collimator may intercept the beam halo, protecting edges of the target, and also stops the back scattered neutrons from target. 


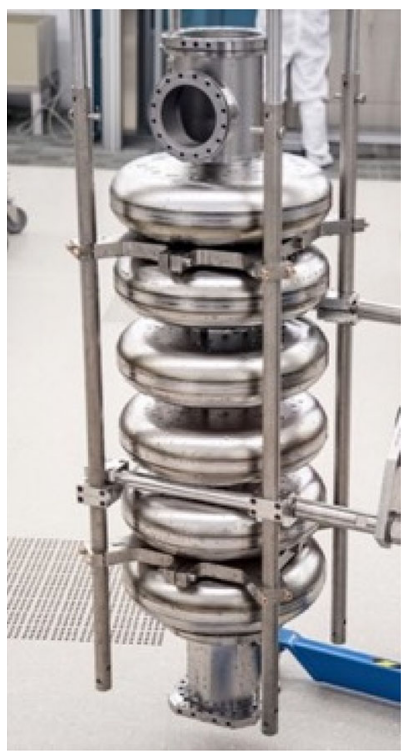

Fig. 5 An ESS medium beta cavity

\section{The working teams and the procurement phase}

The ongoing project at ESS is pioneering in so many ways. It is not only breaking new ground in the equipment and capability of the facility, but also in how the project itself works. Most of the equipment is as a result of In-Kind Contribution (IKC) Agreements, which represent a total of $€ 550$ million, almost one third of the total budget. These collaborations are with over 100 institutions all over the world, in what has and will be a landmark in cooperation within the scientific community.

ESS is a greenfield project, meaning there was no existing template to work within. The partners are not providing only equipment, but they are asked to transfer valuable knowledge. A great deal of coordination is needed to match the bureaucratic organization of different contributors with the ESS ERIC one. The collaboration with industry was considered not as a mere best value for money but sometimes analysis of the industrial capabilities has driven the accelerator design, in order to avoid showstoppers and to permit more competition. Close and efficient collaboration with industry is also an asset to drive technological growth and to strengthen the competitiveness of the industrial systems of the countries involved in the project, i.e. in this sense ESS is a further demonstration of the role of Large Research Infrastructures (LRI) construction as a benefit for the economy. These very specialized commitments, with challenging technical requirements and tight schedule constraints, often pushed several companies to join their forces to meet the project requests. It is in fact strategic to develop a critical mass on projects that require significant investments over short periods, this entails creating alliances that increase production capacity and reduce the risk, to the benefit of both companies and the Public Research Bodies (an ERIC in this case). These temporary joint-ventures are, in fact, effective both for the supply of 
products and for job management. The Italian contribution to the ESS supported this good practice, as it represents a guarantee for respecting execution times: for example in the case that an industrial partner is lacking technical personnel, the partner company can promptly step in. Another advantage is the possibility of sharing know-how as well as infrastructures, such as laboratories, instrumentation and test stands which may require major investments. It is therefore an effective risk mitigation method.

Additionally, the partnership with industries followed the production chain, trying to adapt good industrial practices to the needs of high performance, high reliability of the project. The continuous interaction was in principle a higher cost in terms of manpower, especially for the in-kind contributors, but it has permitted to minimize the manufacturing defects and to keep the level of requirements' fulfillment high. As for the definition of the scope of the in-kind contribution of INFN, CNR and Elettra Sincrotrone Trieste, this was done by the Italian Ministry of Research according to the available expertise at each Institution and INFN was appointed as Representing Entity of Italy in the Europan Spallation Source ERIC, so that the funding was yearly transferred to INFN, that has been taking care of the procurement procedures in synergy with CNR and Elettra. For coordination and information exchange purposes, a Committee with a member of each of the three Institutions filtered all documents and opportunities with the aim to propose the suitable actions to the Presidents of those Institutions and to work as contact team for the ESS ERIC. As for the operational roles, the construction of the components defined in the technical agreements for the in-kind contribution to the Accelerator was defined by INFN and Elettra, while the construction of the Neutron Science instrumentation was done mostly by CNR.

The realization of the technical contribution was organized in Work Packages (WP). The scientific and technical responsibility was assigned to INFN for the WP in charge of the construction of the Source and LEBT (at the Laboratori Nazionali del Sud in Catania), of the Drift Tube Linac (at the Laboratori Nazionali di Legnaro and at INFN-Sezione di Torino), of the Medium Beta Superconducting Cavities (at LASALaboratori Acceleratori e Superconduttività Applicata of INFN-Sezione di Milano); it was assigned to Elettra for the WP in charge of the construction of the RF power stations, the magnet power converters, the magnets and the wire scanners acquisition system for th beam diagnostics (every package was fully managed in Elettra site, except for purchase activities that were carried on in collaboration with INFN). For each WP the responsible and the working team were defined at local site. This permitted to guarantee the best quality, provided that hiring of engineers and technicians with temporary contracts, according to Italian regulations, could be coherent with the time schedule. Unfortunately for INFN the time lapse requested for the hiring process and the large request of similar expertise by industries added up to restrict the available personnel devoted to ESS and the involvement of more staff technicians was necessary to cope with the project schedule. Such a difficulty obliged the INFN units to achieve a higher level of flexibility, in order to produce a recovery plan by mixing skilled staff with newly assigned personnel and by using the former to drive the design and construction phase. The opportunity of training of young researchers and technical staff has been also an important benefit resulting from the activities for the realization of the in-kind contribution. 


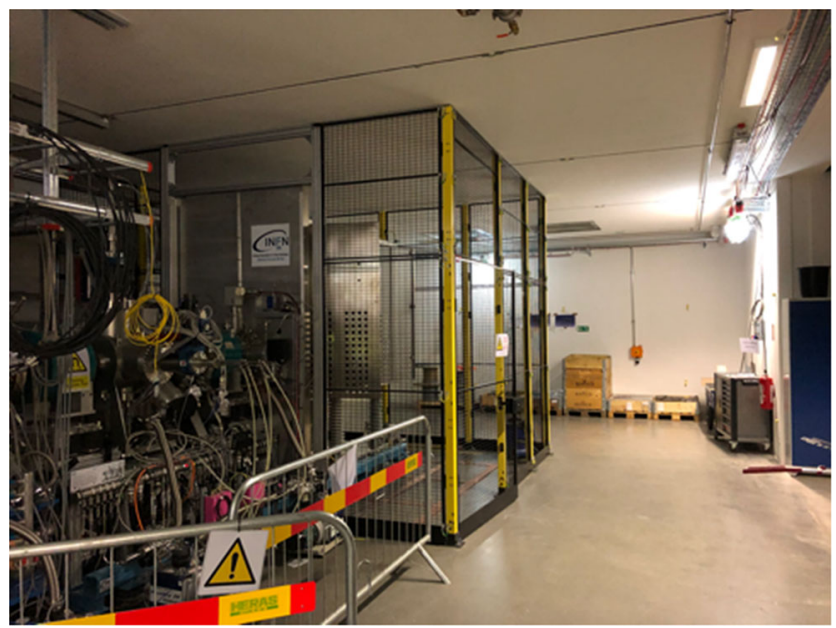

Fig. 6 Ion source and LEBT installed at ESS, Lund

\subsection{Ion source and LEBT}

The proton source for ESS (PS-ESS) is a $2.45 \mathrm{GHz}$ microwave discharge ion source (MDIS) with a very flexible magnetic system able to provide magnetic field from few hundreds to two thousands Gauss. The microwave power is provided by a $2 \mathrm{~kW}$ magnetron and the impedance matching with the plasma chamber is achieved by using a four-stub tuning unit. An MKS flow controller feeds the gas into the OFHC cylindrical plasma chamber to reach a very high level of repeatability, stability and the precision of the injected flux. The insulating column permits to sustain up to $90 \mathrm{kV}$ and the beam extraction was tested up to $130 \mathrm{~mA}$ at the nominal voltage of $75 \mathrm{kV}$ without any sparks. The source is housed in the high voltage platform shown in Fig. 6. These technical choices follow a previous optimization of MDIS that is described in [20]. Specific improvements of PS-ESS with respect to the magnetic field values in order to maximize the energy content of the plasma was favored by an original improvement of the computer control system.

In order to characterize the beam performances in a wide range of parameters a custom high-level code was developed. This code is able to change the value of all source parameters and read the output data of all diagnostic equipment allowing to explore a wide parameter space and define the source settings able to satisfy the ESS requirements. The results satisfied the configuration required by ESS with current above the $70 \mathrm{~mA}$ of proton at the end of the LEBT (Fig. 7). The measured stability was $\pm 1.5 \%$ inside the beam flat top, and $\pm 3 \%$ among pulses [30]. The rise time is about $440 \mathrm{~ns}$ and there is a high repeatability between the produced pulses. The maximum deviation was $\pm 10 \mathrm{~ns}$ collected for one thousand pulses. The gas input system and the extraction column were properly designed to get such stability, that was possible by means of integration between high precision mechanics design and computer controls, mostly done by local industries. 


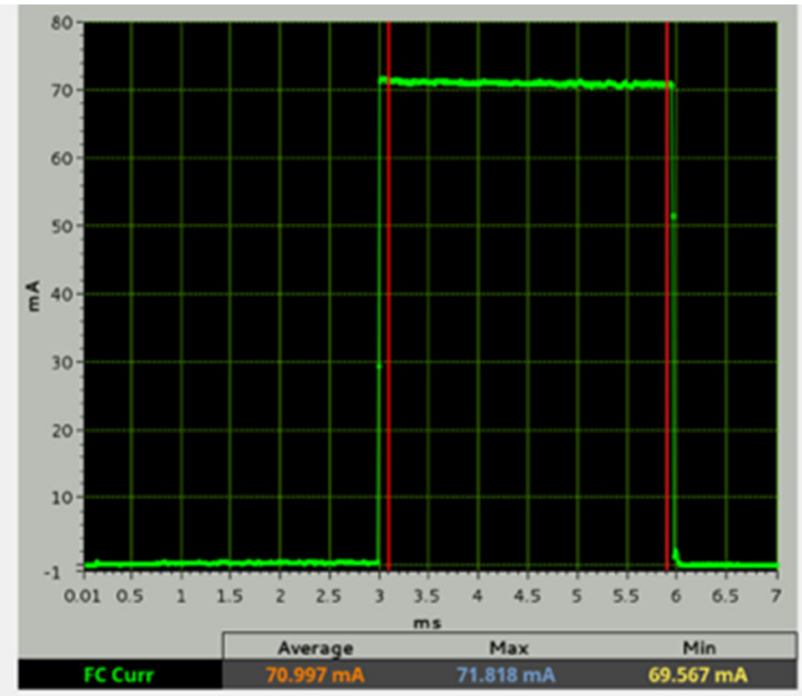

Fig. 7 The proton beam at the end of the LEBT, measured by the Faraday cup

Similarly, the optimization of the LEBT was significantly eased by the collaboration with Italian and French partners. The first element of the LEBT has been designed as compact as possible to minimize the length between the first solenoid and the extraction aperture. This element houses the support of the electrode extraction system, two turbo pumps, three vacuum gauges, a residual gas analyzer, the water feedthrough of the electrode cooling system and the high voltage feedthrough of the repeller electrode. The two solenoids, with embedded X and Y steerers, have been designed in collaboration with CEA. A six movable blades iris was designed to be able to reduce the amount of transmitted current without changing the source settings. The mechanical complexity of this unit, able to stand high power beams, was set by a customized design by INFN engineers and an Italian SME. Before the second solenoid two emittance measurement units (X and Y planes), a Doppler shift measurement unit, two cameras for the beam alignment, two turbo pumps and the chopper were integrated in a short as possible vacuum chamber, to reduce the beam emittance growth. The LEBT ends with a conical collimator that works as a beam dump for the $\mathrm{H}_{2}$ beam produced by the source and the part of the proton beam pulse which is deflected by the chopper. The collimator works also as a differential pressure vacuum sector dividing the accelerator from the LEBT where a pressure of a few $10^{-5}$ mbar is needed to activate the beam space charge compensation and keep the measured emittance below the nominal value of $0.25 \pi \mathrm{mm} . \mathrm{mrad}$. (in Fig. 8 the beam emittance is measured $9 \mathrm{~cm}$ after the RFQ matching point, where the beam is diverging in absence of the accelerating field [30]). All elements here mentioned are installed in about $2.5 \mathrm{~m}$.

The PS-ESS source and LEBT were fully installed and commissioned in Catania prior to the delivery to ESS. The commissioning consisted of two main stages: (1) measurements of beam current, fraction of ion species, and emittance at the exit of the ion source, and (2) beam transport optimization, and emittance measurements in the 


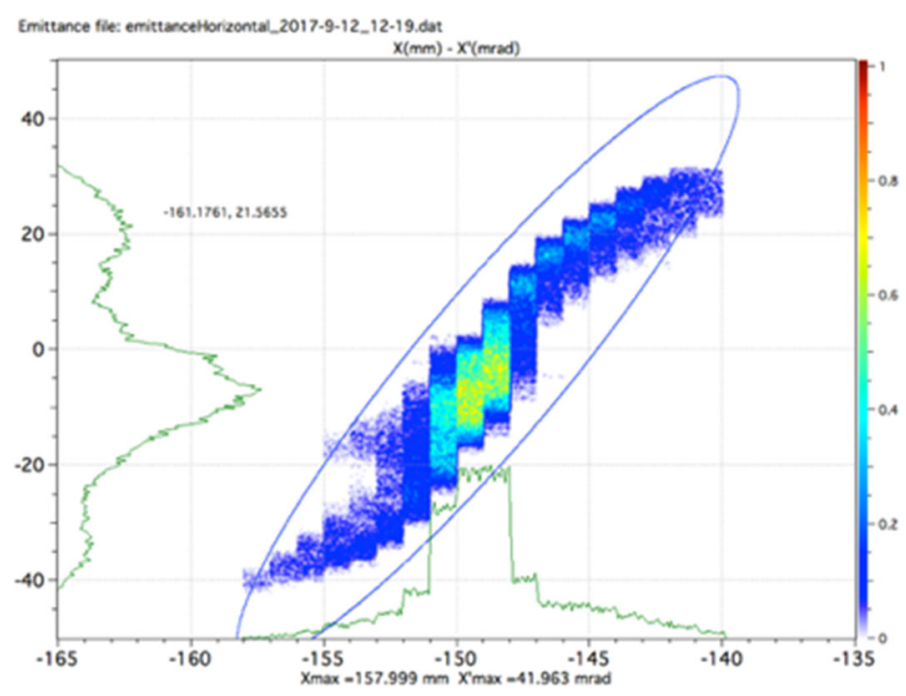

Fig. 8 Ion source beam emittance for $\mathrm{I}=70 \mathrm{~mA}, \varepsilon=0.238 \pi \mathrm{mm} \cdot \mathrm{mrad}($ expected $<0.25 \pi)$

LEBT. The fractions of the different ion species were measured with a Doppler shift monitor. With the nominal ion source settings, the measured fractions were $85 \%, 12 \%$ and $3 \%$ for protons, $\mathrm{H}_{2}{ }^{+}$and $\mathrm{H}_{3}{ }^{+}$respectively. The source produced the nominal time structure of $6 \mathrm{~ms}$ pulse length and $14 \mathrm{~Hz}$ repetition rate, with the first $3 \mathrm{~ms}$ removed by the chopper. The ion source and LEBT produced beams with flat top pulse length in the range of 1-3 ms, without significant changes in the beam current. The repetition rate, varied in the range of $0.25-28 \mathrm{~Hz}$, had a small effect on the beam current measured at the end of the LEBT but no effect on the beam stability.

The disassembly of the Ion Source and LEBT and the packaging was then completed in just 19 days. During the packaging phase all the humidity-sensitive devices have been packed under anaerobic nitrogen atmosphere in order to avoid oxidation of metal part. Dedicated track with air suspension and shock monitoring system was used to transfer the overall setup to Lund. The shock monitoring system consisted of a real time GPS system to monitor and alert the driver if a preset level were breached and equipped of 2 shock, vibration, acceleration and environmental (temperature, humidity and pressure) data loggers and recorders. The unpacking and reassembly has been carried out by the LNS-staff in collaboration with ESS personnel in only 14 working days.

A Safety Readiness Review (SRR) with an external committee was successfully held at ESS in July 2018 with the purpose of providing an independent review of the readiness to safely commission and operate the ion source and LEBT. Following the SRR, all sub-systems were tested and were ready for the final beam commissioning. It can be concluded that the success of the procurement and construction phases of the source and LEBT was originated not only by key scientific actions (definition of plasma parameters, microwave injection, beam dynamics) but also by technological ones and management issues. 


\subsection{Drift tube linac}

The DTL (Drift Tube Linac) is a normal conducting RF structure able to accelerate an intense beam with excellent performances in terms of beam dynamics (beam quality and lossless transmission) and RF efficiency (low power consumption). The focusing lenses (compact permanent magnet quadrupoles) are integrated in the drift tubes [31], together with beam diagnostics and steering dipoles, the RF field is stabilized against time dependent perturbations by a system of quarter wave antennas (post couplers) and the resonant frequency is kept by means of movable tuners [32].

The DTL construction has been proceeding through.

- The procurement of many components in industry.

- Internal INFN work for the most critical parts (drift tubes, brazing, integration of beam components).

- Developing and debugging the bead pull system for accelerating field measurements, including the acquisition algorithm.

- Study and implementation of a field stabilization technique that employs a proper post-couplers setup.

- Verification of the correctness of the chosen DTs face angle values and their effect on frequency and field when post couplers are inserted.

- Preparation for the various integration steps in Italy and at Lund ESS site.

The main points for the management of all the process are to ensure the best quality and to maintain the dates for installation (RFI). A collaboration with CERN and GSI have been established for copper plating, which delayed the starting time of the process but guaranteed the highest quality of plating.

The design, constructions, testing, installation and RF conditioning of the DTL is done by INFN-LNL and INFN-Turin. The 39-m long DTL is designed to accelerate the proton beam from 3.6 to about $90 \mathrm{MeV}$ and operates at a frequency of $352.21 \mathrm{MHz}$. Each of the five DTL tanks consists of 4 modules of $\sim 2 \mathrm{~m}$ length and a tank diameter of $520.5 \mathrm{~mm}$. The tanks are made of AISI 304L stainless steel with copper plated internal surface (Fig. 9). The drift tubes are positioned on a girder - a precisely machined aluminium alloy structure - which is located on top of each module. The drift tube bodies are made of OFE copper, with copper plated AISI 304L stainless steel stems. Stems and drift tubes bodies are vacuum brazed with internal mechanics then the components, such as beam position monitors, steerers, and Permanent Magnet Quadrupoles (PMQs), are inserted and the covers are sealed by electron beam welding. The DTL tanks contain a total of 89 PMQs. The beam trajectory correction is done by 30 steerer magnets installed throughout the DTL. Each tank is equipped with two power couplers to feed the required 2.2 MW peak RF power for operation into the DTL tanks. A total of 123 post couplers have been integrated for field stabilisation during the tuning process. Active tuners control the resonance frequency during operation and tune the field distribution locally. DTL tanks contain 17 Beam Profile Monitors and the intertanks sections contain 5 Beam Current Monitors and 2 F Cups. The machining of the tanks and girders has been done at CINEL in Italy, with daily interactions of INFN experts. The machining of the drift tubes is done at INFN in Legnaro and Torino. The Permanent Magnet Quadrupoles and steerers are delivered to Legnaro and are 

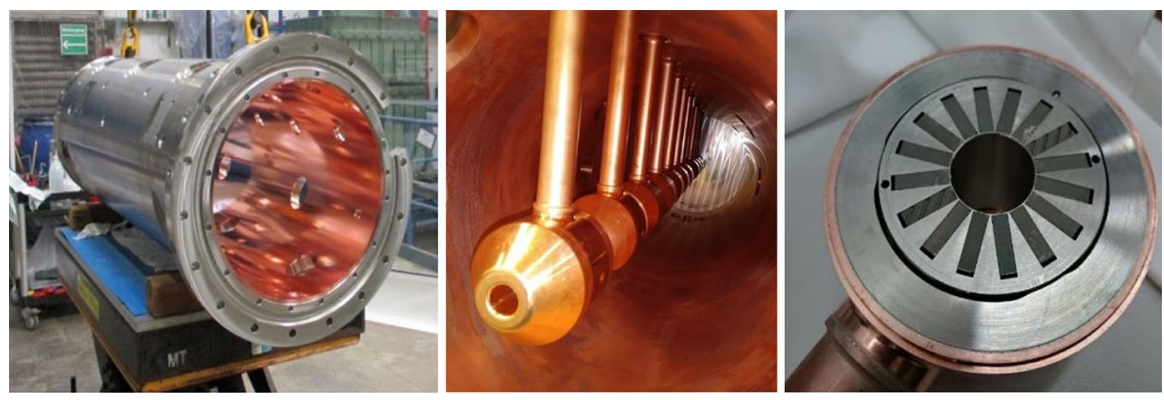

Fig. 9 Tank, drift tubes, permanent magnets during the assembly phase

being assembled into the drift tubes. Brazing is also done at INFN-LNL, while copper plating has been done at CERN and GSI. An aluminium mock-up model of the DTL have been produced by INFN-LNL in order to optimise the tuning procedure and to train the personnel.

The assembly of the DTL tanks is done in a dedicated workshop at ESS. The workshop consists of an enclosure with an HVAC system for temperature regulation and cleanliness, several assembly benches, and an overhead crane. The assembled tank is tuned and placed on a transport trolley, also used to transport it into the tunnel for installation.

The construction of the DTL parts $[31,33]$ is close to completion, while the assembly phase in Lund will take a longer time, until the mid of 2022. The commissioning of the Normal Conducting Linac is expected in June 2022.

It can be seen that the DTL is a complex part of the accelerator either for the scientific requirements (beam dynamics, radiofrequency, diagnostics) but even for the large number of mechanical technologies that are necessary, that required the common work of different INFN experts, of private companies (most of them from Italy but not only) and of other Research Institutions.

\subsection{Medium beta cavities}

Medium beta superconducting cavities construction took benefit of a success case of integration between the skills of INFN, of other European Scientific Institutions and of an Industrial partner, i.e. Zanon, in Schio, Italy [28]. The scheme of operation is shown in the Fig. 10 and followed an analogous strategy as for the XFEL successful experience and prior ones, doing the cold test in the same qualified infrastructure at DESY. The fabrication of 36 cavities for ESS was a series production at the industry, including treatments, tuning and He tank assembly, by following a Built to Print strategy.

Cavities have been delivered at CEA ready for the cryomodule assembly and for the cold test. Delivery rate was expected to reach two cavities each three weeks, but a number of hurdles did not permit to keep this pace. The construction started from the rough material; about 4 tons of $\mathrm{Nb}$ sheets have been transformed after scanning in the different parts. $\mathrm{Nb}$ sheets have been deep drawn to form the half cell, half cells have 


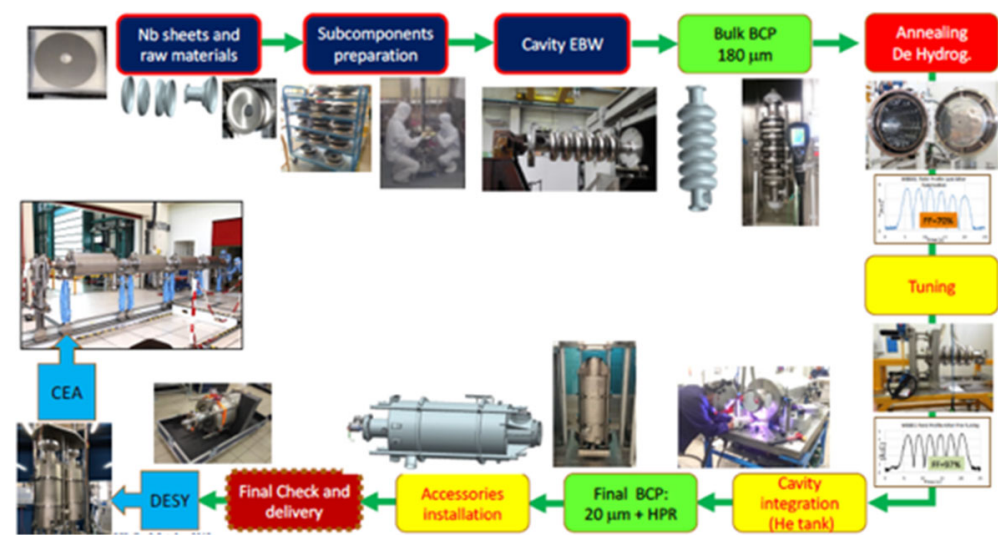

Fig. 10 The production cycle of the medium beta cavities

been electron beam welded to form the dumb bell, then all parts are electron beam welded together to form the cavity. Surface treatment follows, then heat treatment at $600{ }^{\circ} \mathrm{C}$ for Hydrogen degassing complete this phase. Inner surface visual inspection has been followed by field flatness tuning and the He-tank is then welded to the cavity. A buffered chemical polishing is finally performed before the cavity is shipped to DESY for test, which results above specifications are shown in Fig. 11. Whereas a large experience was yet available on the production chain, nevertheless INFN had to improve it with specific research on the prototypes for different reasons:

- INFN design should be plug compatible with ESS cryomodule.

- Specific know how should be transferred to the industry for keeping the same level of performances and minimizing the non-conformities with respect to the ESS requests.

The integration with the company that produced the medium beta cavities was a remarkable element of this in-kind contribution, as they were able to follow the prescriptions of the INFN team because of previous experience with a similar working scheme for the XFEL accelerator [28, 29]. Additionally, in order to ensure the compliance of LASA supplies with the specified requirements and to maintain a Quality Management System according to the EN ISO 9001 a Document Management System (DMS) has been developed for all production documents, such as inspection sheets, specifications, drawings, quality documents, test schedules, inspection procedures, test reports and non-conformity. Alfresco is used to store all documents. A Java application, that runs every $15 \mathrm{~min}$, synchronizes data between Alfresco and the MySQL database. Finally, a powerful dashboard shows the status and the overview of the production.

\subsection{RF power stations}

The cost for a linear accelerator is dominated by the cost for the Radiofrequency system. The RF system also takes more space in the dedicated RF gallery than the accelerator in the tunnel. ESS will use individual RF tubes for all the superconducting 


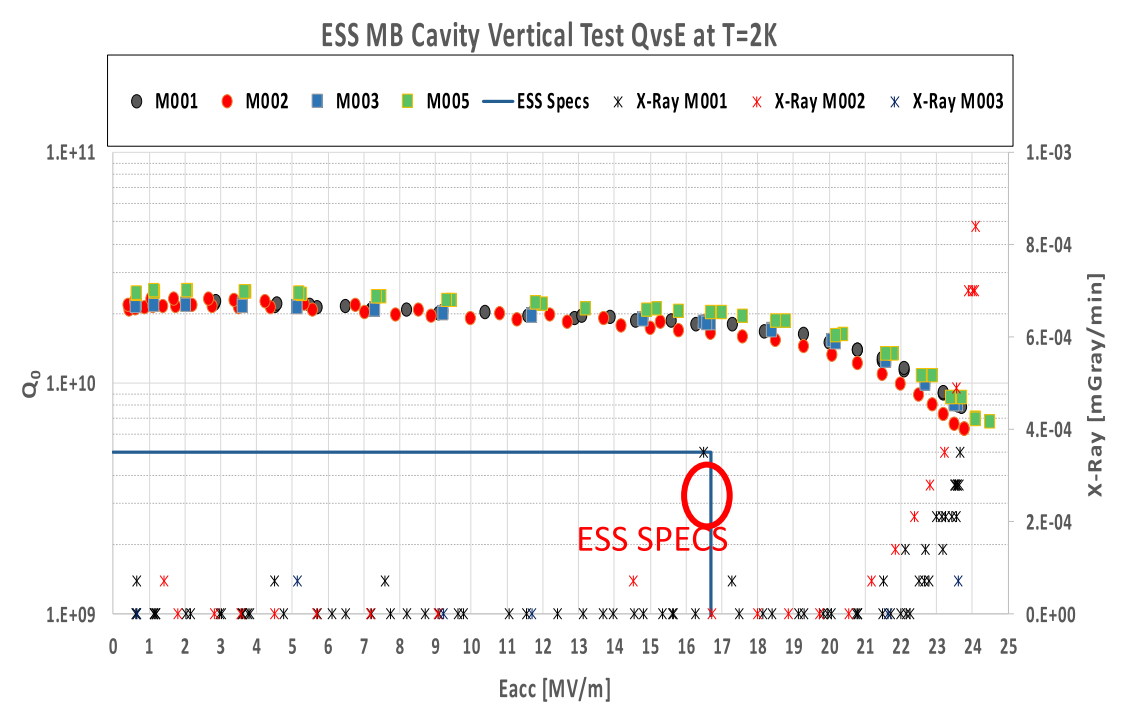

Fig. 11 Test results for one of the ESS medium beta cavities

cavities and larger tubes to drive the different normal conducting structures [1]. The Low Level RF (LLRF) system for the $704 \mathrm{MHz}$ RF systems is designed at Lund University and built by three Polish universities and institutes forming the Polish Electronic group. ESS Bilbao contributes with turn key RF sources for the normal conducting Linac RF systems and control design and construction for all $352 \mathrm{MHz}$ RF systems. As mentioned earlier, Elettra in Italy contributes with tetrode based RF systems for the spoke amplifiers. Twenty-six RF power stations (RFPS) will provide the RF power for the spoke cavities installed in the ESS linac. A one source per cavity scheme is adopted to allow the independent setting of RF amplitude and phase. The RF power requirements range from 260 to $330 \mathrm{~kW}$ pulsed at $352.21 \mathrm{MHz}$ depending on the cavity position along the linac. Considering the margins required by the LLRF regulation and taking into account RF transport losses, the specification of the RFPS have ben standardized to $400 \mathrm{~kW}$ peak nominal, with a $5 \%$ duty cycle. RF pulse duration is equal to $3.5 \mathrm{~ms}$ and the repetition frequency is $14 \mathrm{~Hz}$. Main specifications are reported in Table 1 [34].

Following a detailed analysis of the alternatives for the power source available on the market, the choice of using tetrodes for the RFPS baseline design has been adopted [35], since it provides performance and costs optimization, as well as an overall simplification of the equipment.

Each RF power station [36] will be realized combining two equivalent transmitters with a $3 \mathrm{~dB}$ hybrid combiner. The RF amplification chain of each transmitter is composed of a $7 \mathrm{~kW}$ pre-amplifier followed by a $210 \mathrm{~kW}$ tetrode as main amplification stage, see Fig. 12. The pre-amplifier is a solid state transistor based, water cooled amplifier. For the main amplification stage, the tube adopted is a tetrode TH595A from Thales. This tube is capable of providing $210 \mathrm{~kW}$ at the required frequency and duty 
Table 1 RFPS main parameters

\begin{tabular}{ll}
\hline Parameter & Value \\
\hline Central frequency & $352.21 \mathrm{MHz}$ \\
Nominal peak power & $400 \mathrm{~kW}$ \\
Gain & $76 \mathrm{~dB}$ \\
RF drive for maximum power & $10 \mathrm{dBm}$ \\
Bandwidth & $>2 \mathrm{MHz}$ \\
Operation mode & Pulsed \\
Duty cycle & $5 \%$ \\
Repetition frequency & $14 \mathrm{~Hz}$ \\
Pulsed width & $3.5 \mathrm{~ms}$
\end{tabular}

Cooling

Forced air and water

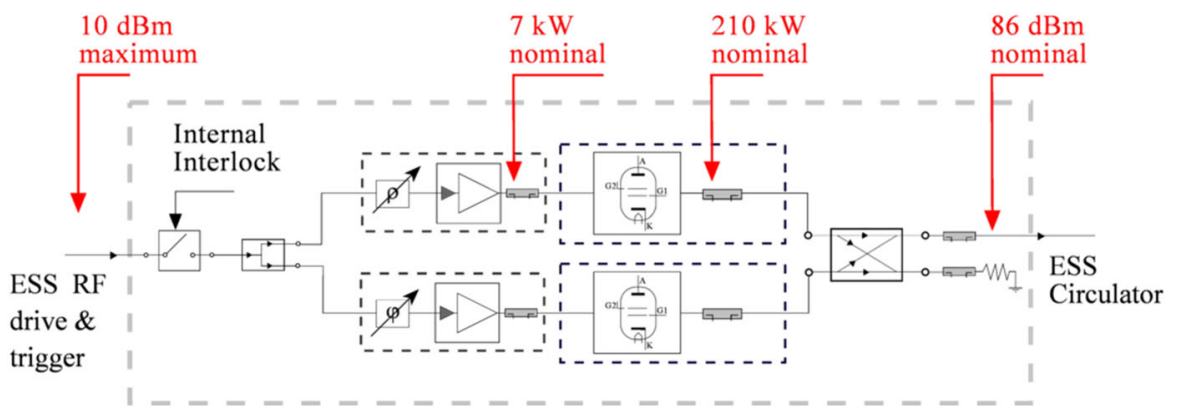

Fig. 12 RFPS amplification chain

cycle once installed in a TH18595A RF cavity. The two tubes are finally combined to reach $400 \mathrm{~kW}$ peak at the output of the station.

The design choices for the RFPS [36] took into account, in addition to the tight specifications of stability and reproducibility of the RF pulse, the fundamental requirement of high reliability needed for a user facility as ESS and of high efficiency, that is to minimize power consumption, addressing one of the basic challenges of a sustainable machine. For these reasons, starting from the beginning, RAMI (Reliability, Availability, Maintainability and Inspectability) concepts have been incorporated in the design. As for the tetrodes power supplies, in each station the two tetrodes share a common HV modulator for the anodes, while heaters and grids of each tube have their own power supplies for independent regulation. In particular the modulator [37] has been designed to provide high voltage stability regulation and high reproducibility. Electrical grid low frequency harmonics and flicker reduction are also important aspects that must be taken into account in the design, considering the high power pulsed operation. For this reasons, the topology is based on a commercial HV capacitor charger which charges a capacitor bank. The capacitor charger was specified to have output voltage and current regulation and limitation capabilities. This design also effectively minimizes power consumption, as requested for environmental and economic reasons. A key aspect of the project is the interlock and control system, that 


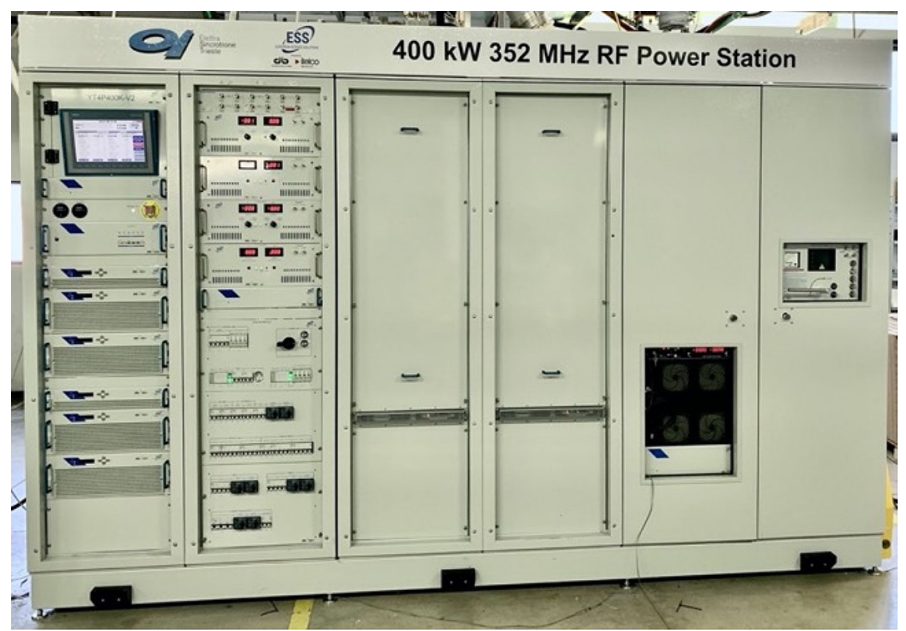

Fig. 13 One $400 \mathrm{~kW}$ RF power station

allows to operate the machine, locally and remotely, in full compliance to personnel, machine and equipment safety.

The integration in the ESS environment poses additional challenges to the project, due the boundary conditions of the space available in the layout of the ESS gallery, which drives high compactness needs, and the importance to assure a smooth and efficient installation and connection to the other systems, as controls and conventional facilities. Modularity and easy access to any component for maintenance purposes are also key design elements. All these considerations, supported also by prototype and laboratory measurement, were included in the technical specifications. The construction of the RFPS has been assigned to European Science Solutions, Italy, a company specifically formed by DB Science and Itelco, major players in broadcasting industries with already a considerable experience in providing high power RF equipment to the scientific market, with the scope to join the effort and exploit the experience and know-how of both the partners. The result is the construction of an equipment that, in addition to the required technical features, has a series of advanced innovations both in equipment management and diagnostics. The design has been validated with the successful completion of the factory and site acceptance test of the first station (see Fig. 13), the latter performed at the ESS test stand in Lund. The construction of the series is now in progress and the plan is to complete the construction and the delivery to Lund at the end of 2021.

\subsection{Diagnostics}

The "eyes" in the operating accelerator for the engineers and physicists working with control and optimization is the beam instrumentation. ESS has a good set of instrumentation starting with Faraday cups, emittance measurements units and a Doppler shift measurement systems for beam type identification in the low energy beam trans- 
port. Beam position, profile and current are also measured in the medium energy beam transport and in the drift tube linac. Along the high energy linac it is also possible to measure profile and emittance using wire scanners. The beam position and beam loss are of course also measured in the SC linac with the beam position monitors serving as phase measurement units. The beam phase advance must be measured to optimize the beam longitudinally. Finally, at the target the beam position and shape is measured. The shape is measured through a scintillating coating on the target itself, which can be observed with a camera over an advanced optical mirror system. The Italian contributions for the beam diagnostics include the electronics for the wire scanners system and the stripline detectors for beam position measurements in the DTL.

Wire scanners are diagnostics equipment used for acquiring the transverse beam profile at different location in the linac. The basic principle of operation is that the system acquires the electrical or optical signal produced when a thin metal wire is scanned across the proton beam. Since the amplitude of this signal is proportional to the beam charge density, the beam transverse profile may be obtained by plotting the signal amplitude versus the wire transverse position. The total number of wire scanner stations to be acquired is eleven. The acquisition system for the wire scanners, that designed and provided by Elettra, is composed of custom developed hardware (HW) modules, linked together by means of an EPICS control and processing software which is also part of the contribution [38].

The HW modules amount to thirteen Analogue Front End (AFE), nine Optical Front End (OFE) and to the associated twenty-two Back-End (BE) modules. The purpose of the analog front end is to read out the current from the single wire intercepting the beam. The analog front-ends (Fig. 14) are located in the accelerator tunnel to minimize the signal loss due to the cable length. Each one of them is connected to the associated back-end module which is installed in the service gallery. The analog front-end is provided with a two channel input stage to cover the full dynamic range.

The purpose of the optical front ends (Fig. 15) is to acquire the light generated in the scintillator detectors, located down-stream the wire scanner, and to convert the light pulses in electrical signals. The optical front ends will be located in the service gallery connected with fiber optics to the equipment in the tunnel. The optical front ends are as well connected to the associated back-end modules.

For this contribution on wire scanner acquisition system, Elettra is also in charge to develop the control and processing software, running under EPICS. This is composed of a set of tabs, known as Engineering Panels, and of the User panel. The control software is interfaced on one side to the back-ends and on the other side to the ESS Motion Controller, which drives the mechanical motion of the wire scanner. Finally, also the design of the cable system for the wire scanner acquisition system is included in Elettra scope.

At the time this paper is written, the hardware construction is completed, both analog and optical front end have passed the factory acceptance test and delivered to ESS in Lund. For the Analog front end also the site acceptance test is completed and the installation in the machine is going on. The site acceptance test of the optical front end is planned soon. It must be acknowledged that a fundamental role for the development of the system has been played by the possibility of testing and validation campaigns in a real accelerator environment, as the ones performed at CERN on Linac4 and at 


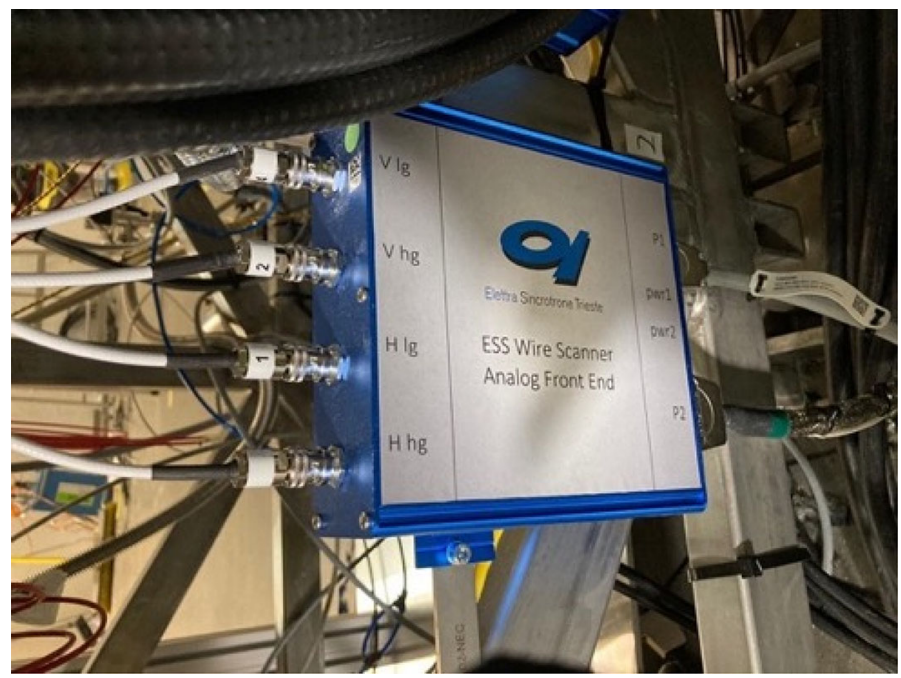

Fig. 14 One wire scanner analog front end module installed in the ESS linac

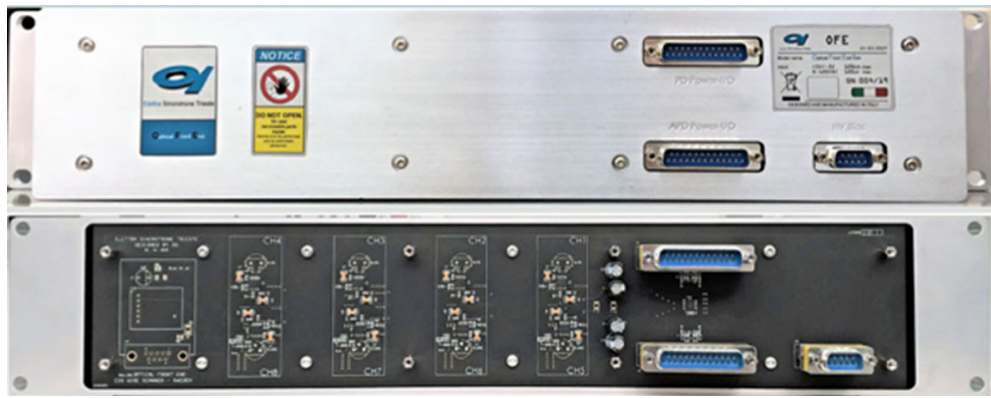

Fig. 15 Optical front end

Forschungszentrum Jülich, and by the vertical integration tests at ESS Bilbao and at ESS Lund.

\subsection{Magnets}

Elettra in-kind contribution scope for the magnets includes the supply of several conventional iron dominated magnets which are installed in the superconducting part of the linac and in the transport lines to the target and to beam dump [39]. In summary this means a total number of 213 magnets of different topology, i.e. 139 quadrupoles, 2 dipoles and 72 correctors, as described in Table 2. All magnets are operated in DC mode.

For all the magnets, the first activity performed was the analysis of magnet performance needed to satisfy the beam optics requirements. This was followed by the design and optimization of the magnetic models, as well as by the definition of the 
Table 2 List of the magnets included in the scope of Elettra in-kind contribution

\begin{tabular}{llc}
\hline Type & Description & Quantity \\
\hline Q5 & Quadrupole for spoke linac & 26 \\
C5 & Dual-plane corrector for spoke linac & 13 \\
Q6 & Quadrupole for medium-beta linac, high-beta linac, high energy beam transport and & 95 \\
& dump line & 55 \\
C6 & Dual-plane corrector for medium-beta linac, high-beta linac, high energy beam & 12 \\
& transport and dump line & 2 \\
Q7 & Quadrupole magnet for accelerator to target ramp & 6 \\
D1 & Vertical dipole magnet for high energy beam transport and accelerator to target ramp \\
Q8 & Quadrupole magnet for accelerator to target & 4 \\
C8 & Dual-plane corrector for accelerator to target & \\
\hline
\end{tabular}

mechanical ones. Specific attention was dedicated during the development phase to standardization and to optimization of the magnets, also considering the impact that the design choices have on the current and power requirements of the power converters. This optimization work has also allowed to efficiently split the procurement in three contracts: one for the Q5, Q6 and Q7 quadrupoles, the second for the C5 and C6 correctors, both assigned to Danfysik, and the third for the remaining magnets, assigned to Sigmaphi. This splitting also allowed an efficient follow-up of the construction, verification and validation processes.

The quadrupoles Q5, Q6 (Fig. 16) and Q7 are water cooled, while the correctors C5 and C6 are air cooled. After the construction, these magnets were delivered to Elettra, where the magnetic measurements and complete characterization took place. For this purpose, a dedicated state-of-the art magnetic measurement lab was developed at Elettra with a new measurement bench, employing rotating coils made in collaboration with CERN. After the completion of magnetic measurements and successful validation, all the magnets were shipped to STFC in UK for the installation on the girders and integration in the linac warm units. As mentioned above, principle of standardization were adopted as much as possible. For the design of these quadrupoles, the same conductor cross-section size for the coils as well the same maximum current density were adopted. Since the magnets Q6 and Q7 share the same bore diameter $(112 \mathrm{~mm})$ and good field region requirements, they were designed to have the same 2D magnetic design, with the same lamination and the same ampere turns. Therefore, the only main difference is in the yoke/magnetic length. The magnetic length of Q6 is $278 \mathrm{~mm}$ while for Q7 it is $338 \mathrm{~mm}$. The Q5 magnets are smaller, having $68 \mathrm{~mm}$ bore diameter and $201 \mathrm{~mm}$ magnetic length. C5 and C6 correctors are window frame combined horizontal and vertical magnets, with pole shape designed to minimize the sextupole component. Also, in this case, to use the same type of power converter, for the coils, the same conductor cross-section and the same maximum current density were included in the design driving. The effect of the corrector installation near the quadrupoles, as it is foreseen for example in the linac warm units, where a corrector is 


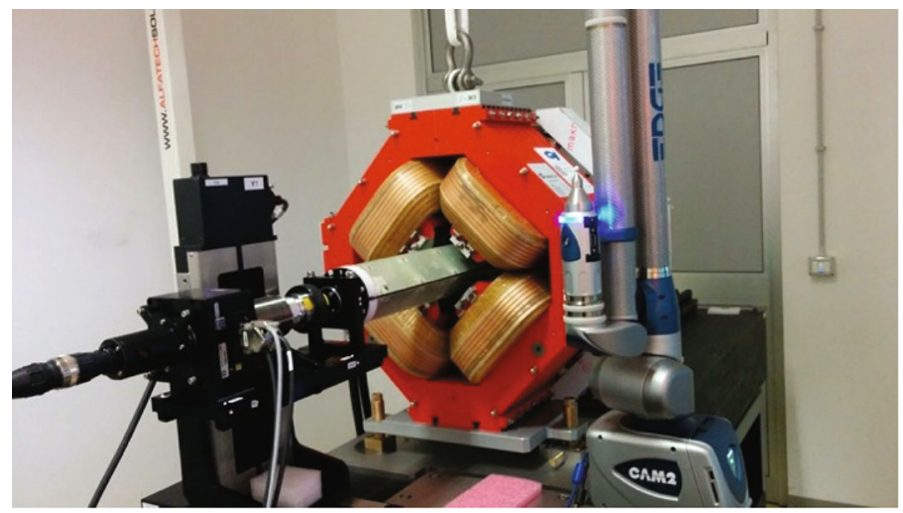

Fig. 16 One of the Q6 magnets on the measurement bench at Elettra

installed between two quadrupoles, was also studied and found fully compatible with the required specifications.

For the magnets of the accelerator to target ramp, i.e. D1, Q8 and C8, Elettra has developed the magnetic models and the basic mechanical models. These magnets have large aperture to accommodate the vacuum chamber. For this reason, special attention has been payed to the ampere-turns calculations. In the case of D1 and Q8 the maximum current of $400 \mathrm{~A}$ has been defined with the purpose to use power converters, identical to those used for Q5, Q6, and Q7, in parallel configuration. The D1 (Fig. 17) and Q8 magnets are water cooled. The design effort has also aimed to keep the overall dimensions of the yoke of such magnets in a reasonable range, for example for D1 this resulted in $1800 \mathrm{~mm}$. C8 correctors are air cooled window frame combined horizontal/vertical magnets. The D1, Q8 and C8 magnets were measured at the manufacturer premises and sent directly to Lund.

All the magnets included in the scope of the in-kind contribution has been built and delivered. They are being installed in the tunnel according to the ESS installation plan.

\subsection{Power converters}

Elettra is in charge of providing all the power converters for the magnets contributed to ESS [40]. Each magnet is individually powered by its own power converter, with the exception of the two dipoles which are connected in series and therefore powered by the same device. It must be also noted that since the correctors are dual plane, they require two power converters. Table 3 reports the full list of power converters. Development of magnets and of the related power converters were performed in parallel according to a common strategy, considering them as a unique system. The resulting benefit of this design methodology is to achieve a high standardization, for both magnets and power converters, and a high modularity for the power converters, both for the DC ones, for the quadrupoles and the dipoles, and for the 4-Quadrants (4-Q) ones for the correctors. This is of course a clear advantage already in the construction phase and 


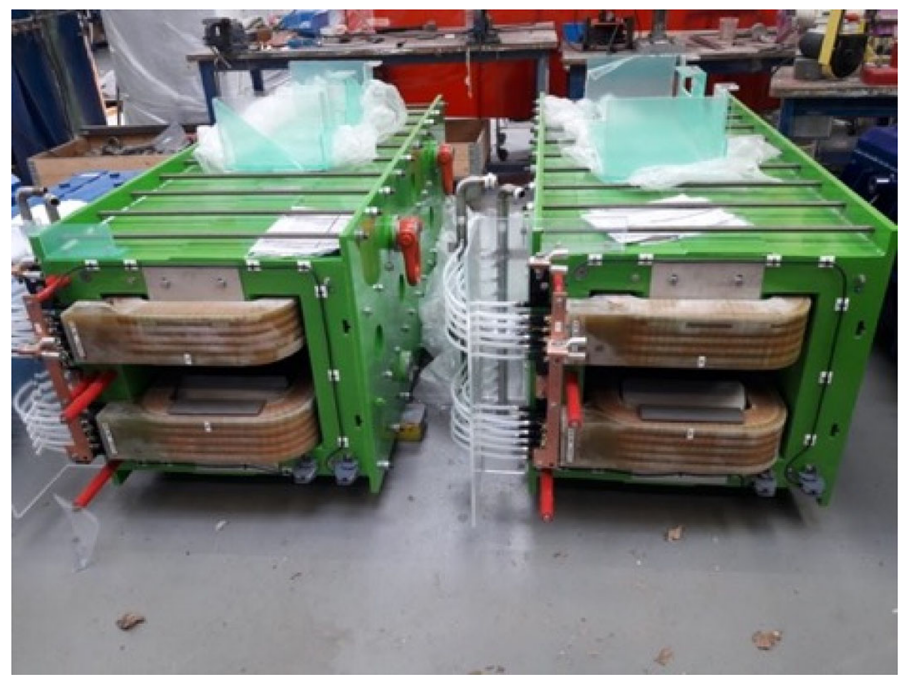

Fig. 17 The two dipole magnets

Table 3 List of power converters

\begin{tabular}{llll}
\hline Name & Magnet & Operating mode & Quantity \\
\hline PCQ5 & Q5 quadrupoles & DC -200 A/50 V & 26 \\
PCQ6 & Q6 quadrupoles & DC $-200 \mathrm{~A} / 50 \mathrm{~V}$ & 95 \\
PCQ7 & Q7 quadrupoles & DC $-200 \mathrm{~A} / 50 \mathrm{~V}$ & 12 \\
PCQ8 & Q8 quadrupoles & DC $-400 \mathrm{~A} / 50 \mathrm{~V}$ & 6 \\
PCD1 & D1 dipoles & DC $-400 \mathrm{~A} / 100 \mathrm{~V}$ & 1 \\
PCC5 & C5 correctors & 4-Q $- \pm 16 \mathrm{~A} / \pm 20 \mathrm{~V}$ & 26 \\
PCC6 & C6 correctors & 4-Q $- \pm 16 \mathrm{~A} / \pm 20 \mathrm{~V}$ & 110 \\
PCC8 & C8 correctors & 4-Q $- \pm 16 \mathrm{~A} / \pm 20 \mathrm{~V}$ & 8 \\
\hline
\end{tabular}

installation, but even more in the future operation for the maintenance and spare parts management.

The optimization of the design has led, in fact, to standardize the specifications for the power converters for the three quadrupoles families PCQ5, PCQ6 and PCQ7 to the same $200 \mathrm{~A}, 50 \mathrm{~V}$ parameters. For the remaining quadrupole family, PCQ8, the specifications are $400 \mathrm{~A}, 50 \mathrm{~V}$, while the two series-connected dipole magnets $\mathrm{D} 1$ are powered by a common power converter which provides $400 \mathrm{~A}$ at $100 \mathrm{~V}$. The power converters for the quadrupoles (Fig. 18) and dipoles are based on industrial products and have been realized by a consortium between two Italian companies, Energy Technology (OCEM) and CAEN. The solution they implemented, based on their standard NGPS model with minor modifications, met the targets of performance, standardization and modular approach. In fact, the power converters PCQ5, PCQ6, PCQ7 and PCQ8 adopt the same 200 A/50 V unit: directly for PCQ5, PCQ6, and PCQ7, with parallel connection of two of the same modules for PCQ8. PCD1 is based on four $100 \mathrm{~A} / 100 \mathrm{~V}$ modules combined in a parallel connection. The parallel connection 


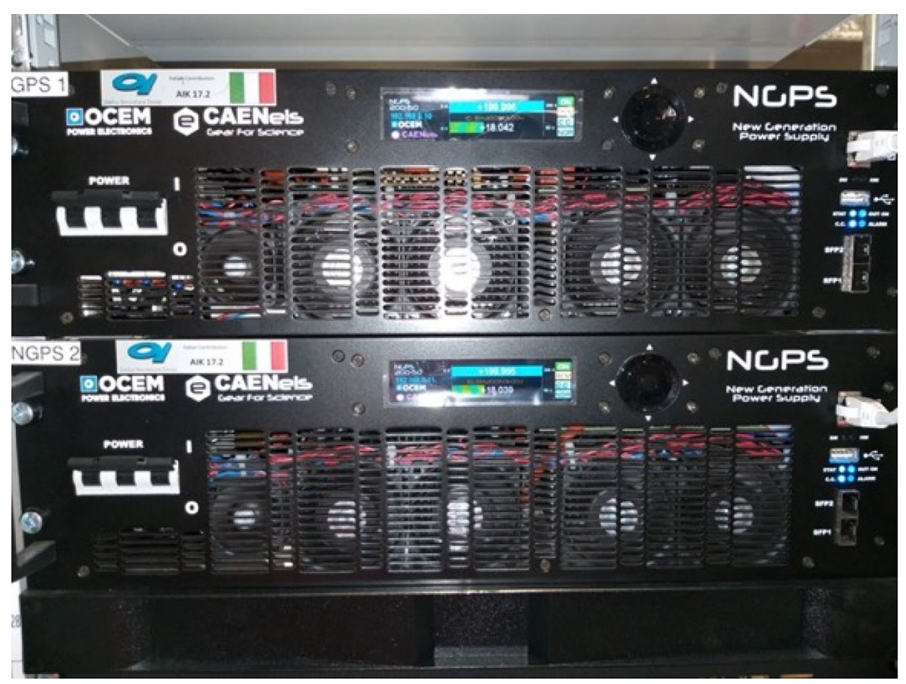

Fig. 18 Quadrupole power converters

of the modules follows a standard "Master $+\mathrm{n}$ Slaves" scheme, and is achieved via optical fibers. For the dipole magnets power converter, an external polarity switch, directly managed by the "Master Unit", has been implemented in the same rack with the four units, to allow reversing the current flow in the D1 magnets to achieve the complete de-gaussing of the dipoles. All the power converters for the quadrupoles and dipoles have been built in full compliance with the agreed schedule and specifications, successfully factory tested and delivered to Lund, where they also successfully passed the site acceptance test.

For the corrector magnets, the design optimization resulted in standardizing all the power converters to the same requirements, i.e. $\pm 16 \mathrm{~A}, \pm 20 \mathrm{~A}$. These parameters allowed to base the design on the successful power converters already designed at Elettra for the FERMI FEL with minor improvements addressing in particular efficiency and reliability aspects. These new, 4-quadrant, $400 \mathrm{~W}$ power converters, named "A2720", have a better efficiency that allows natural air convection for cooling, and also removes the need of fans, which are typically one of the major sources of failures or periodic maintenance needs in similar equipment. A typical $3 \mathrm{U}$ crate hosts four independent power converters (Fig. 19). The construction of units and crates has been assigned to the company EEI, according to a built to print strategy, and is now completed. The units were then delivered to Elettra for firmware uploading, calibration and full testing. All the power converters for the correctors have successfully tested and delivered to Lund. Following the completion of deliveries, the site acceptance test was performed at ESS. All the power converters (for dipoles, quadrupoles and correctors) are now being loaded in the racks and deployed according to the ESS installation schedule. 

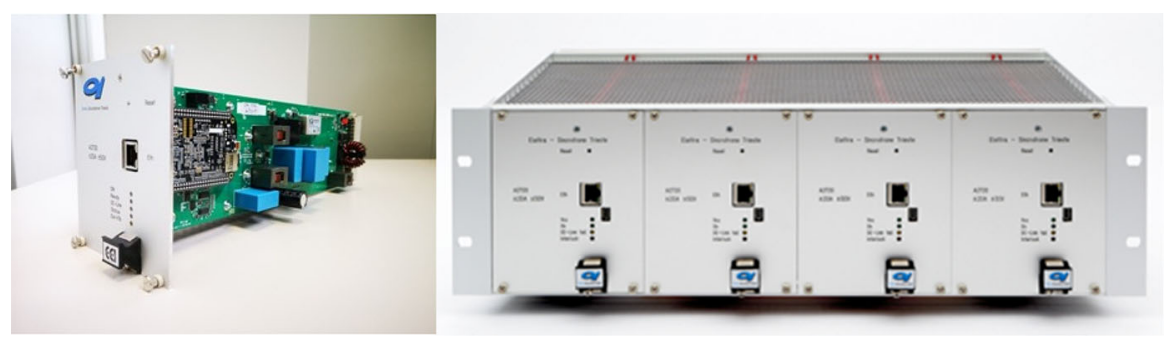

Fig. 19 One A2720 power converter and a 3U crate hosting four A2720 units

\section{The lesson learnt}

\subsection{Technical issues}

Following previous experiences, the contributions of INFN and Elettra to the construction of the accelerator for ESS were easily defined and the Institutional agreements followed, with the scope to transfer to ESS staff the best hardware and know-how for each component of the machine, according to the principles of reliability and sustainability, so that the ESS staff could manage each component without the support of the in-kind contributor. The opportunity of contributing to the ESS construction was felt also as a main advantage for the Institutions, because of the challenges embedded in the design, that could stimulate the local teams to get further skills and to hire and train younger experts to cope with these challenges.

In the organization model of the Italian contribution to the ESS Linac, each Work Unit is responsible for the Industrial Partners qualification and supervises the construction and the Quality Assurance and Quality Control processes during the manufacturing, by implementing check procedures already before the calls for tender and during the construction phase, i.e.:

- detailed schedule for production and delivery;

- scheduled meetings with ESS Accelerator team;

- design reviews at various stages of the project involving also independent external technical experts to provide an independent review of the design;

- periodic meeting with companies and scientific partners;

- factory and site acceptance tests:

- actions in situ in case of problems and/or non-conformity events.

The industrial partners not only integrated very well their skills with the ones of Public Research Institutions but they also increased their capacity to adapt to challenges at the cutting edge of the technology. In any of these cases the industrial partners have been obliged to improve the quality of their products and of their production organization to fulfil the challenging requirements, which resulted in a further advantage for them in other tenders out of ESS.

The challenge was better faced in some cases with the partnerships of two or more Italian companies providing complementary know-how, thus strengthening each of them, an asset especially important for SMEs, and at the same time an evident gain for 
the Italian ecosystem. An example was the association of companies for the largest call for tender of the Italian in-kind contributions, the RF power stations (RFPS). The joint venture of two leading companies in the broadcasting and scientific field (DB Elettronica Telecomunicazioni and Itelco Broadcast), named ESS (European Science Solutions), both playing a leading role in the Radio Frequency domain regarding science and medicine, with over forty years of experience that included similar RFPS, permitted to make a successful proposal. The manufacture of 26 RFPS stations providing $400 \mathrm{~kW}$ output power with mixed technologies (Solid State and Tetrodes), is not easily achievable since it is a prerogative of those who have rooted knowledges in the past and who use advanced technologies in the present. The design of the stations required also a technology advancement and the interaction with the experts at Elettra and ESS played an instrumental role to meet the demanding requirements. It was also estimated that a single company could not deliver the requested number of stations in the scheduled time lapse and the association obeyed to this evidence. The companies, anyway, are competitors in other sectors such as Radio and TV broadcast and even in scientific and medical world, so achieving harmony in the joint venture has not been easy. They had to overcome the inevitable mistrust and the company's individualism but this time the awareness of a common convenience pushed them in the right direction.

A second case, with a different collaboration scheme between the partners, was the one related to the power converters. In this case OCEM and CAEN are somewhat different and complementary. Their partnership, already in place for similar projects, allowed to optimally exploit the best of their individual consolidated know-how and based on this they develop an equipment reaching the highest quality and performance standards. In addition their partnership organization permitted to cover the project requirements with a very high production rate, keeping the highest quality standards.

In summary It can be said that the consortium of two competitors brings to:

- optimization and sharing of technologies;

- better management of production capabilities;

- meeting the required production rate;

- diversify the production on different markets by coping with future market oscillation;

- set the basis for future alliances and reaching the critical mass needed in the market;

- not being invasive on the turnover of the company (it is obvious that a company cannot base its $100 \%$ turnover for years on a single project which then finishes).

In addition the companies are learning a lot from the work they have done for ESS and the effort that the teams in Italy and also at Lund are dedicating to transfer knowhow. Particularly they had the opportunity in some cases to improve their technology, better the QA/QC and supply chain management, implementing lesson learnt, etc. to meet the required standards. This experience will be also an essential reference to qualify them for future large research infrastructure projects.

If we look from the point of view of Institutions, the technical advantages that have been obtained from the mix of Public Research Institutions know-how were really valuable because often they completed each other, with similar complementarity as it was for companies; the strategic alliances may be continued in the future. 
The work was subject to a review in terms of long term sustainability, that is not a trivial issue, as it obliged to increase the documentation to be transferred and in turns to increase the number of internal reviews of the provided contributions that are expected to meet the goals of high reliability, high availability, easy maintainability and inspectability set by the project. For each component a shared folder was provided to ESS ERIC to favor an efficient management and storage of the documents, collecting:

- Drawings

- Diagrams

- Cable specifications

- Manuals

- Factory acceptance test

- Guides

- Interface drawings

- Certification of conformities

- Non conformity reports

- Spare part lists.

This process is also fundamental to meet the scope that ESS has full technical ownership of each part.

\subsection{Social and economy issues}

In Europe a relevant part of the wealth is coming from high tech companies, often developing for the consumer market ideas and products coming from research centers with a low technology readiness level (1 to 4$)$. The supply-chain of cutting-edge technologies is an opportunity for the industrial world; companies that have invested in working on prototypes for research are subsequently acquiring other important orders for similar technologies. More generally, the participation of SMEs to the development of a large research infrastructure improves their competitiveness and stimulates a continuous upgrade of their facilities and of the skills of the workers.

ESS is an incredible challenge for companies and research institutions, consolidating the synergies and strengthening the production chains. The interaction among international teams originated process and product innovation, seeding new production as a consequence of the research base. The participation to each call for tender obliged to invest in know-how, while the procurement phase pushed to invest in new instrumentation, which in turns generated a higher capability to get further orders with high added value, even in different technological field (e.g. some of the Italian companies involved in ESS tenders are active in fields like automotive, microelectronics or oil extraction). Even for the cases of company not successful in the tenders, the work done to fulfill the specifications has been a remarkable asset in future activities and sometimes we observed that the company that was runner up was successful for a next opportunity. In average, all those who applied for these challenges got an advantage.

The social and economic impact of whatsoever Research infrastructure is well established and it is subject of studies for economists, outside of the scope of this paper [e.g. 41,42]. But it is useful to remark that the ESS experience is absolutely innovative from this point of view, having organized a distributed procurement without 
a hierarchical setup, but with a truly democratic process based on the best available know-how among the partners. Multicenter definition of the goals made longer the Accelerator Design Update phase but it permitted to emphasize the risk analysis phase, in order to transform a big challenge in a Project with reasonable parameters for each part of the machine. From the point of view of the technological impact, the presence of many contributors in different nations permitted to spread the information and to involve more companies than it would have been with a pyramidal organization, that may have limited information about the available technologies or may suffer of unconscious bias towards a small number of companies involved with the personal experience of the managers in previous projects and activities.

\subsection{The ESS perspective}

While this paper focuses on the Italian contribution to the ESS accelerator construction project, we also want to briefly discuss some aspects of the ESS central perspective of running such a large collaborative project. The central ESS organization as well as the infrastructure for ESS was built up in parallel with the design and construction work at the IK labs. The initial work to update the conceptual accelerator design and to create functional requirements for the many IK contributions needed was done in close collaboration with the institutes and universities aspiring to contribute as IK partners. The central organization was initially very small and has only approached numbers resembling other national and international neutron sources recently. Consequently, the transfer of knowledge to staff in Lund, the support and manpower available in Lund for installation, testing and commissioning work have been and remain challenging. The future operation of the facility with very few in the central organization having been part of the teams who designed and built the accelerator will also be a major challenge. Only a good and strong continued link to the IK partners will enable ESS to successfully transfer from a construction project to an operational neutron source. The exact nature of that link is still to be determined but it is evident that the many strong personal links between the people in Lund and at the IK partners built over the years of design and construction are an important part of it. These links are an important asset for each Institution, to be used for future projects.

\section{Conclusions}

ESS is the second largest European project in the field of accelerators, after LHC. It is therefore expected an important impact in terms of generating value for the industrial ecosystem. ESS is a social, political, economic challenge, not only a technical and scientific one and it may be a useful example to the deployment of future Research Infrastructures. The in-kind contribution of Italian Public Research Institution to the European Spallation Source was considered since the beginning an opportunity to offer precious know-how getting back an equally precious experience to make stronger the Italian innovation system consisting of Companies, Research Institution and Public Administration. 
The present phase of European development needs that similar activities are following a real change of paradigm, above the level of scientific and technological chauvinism. It can be said that there is no alternative to the collaboration of the best teams to achieve the best results, and none can realistically do it by means of local solution. The concerns for the future are involving even the sustainability of Research Infrastructures that needs a broader and long term vision. In conclusion, for the socioeconomic development the solution is found in the Research, and good Research can be done only by partnership without walls and curtains.

Acknowledgements The authors are grateful to the colleagues working for the in-kind contribution to the ESS Linac in INFN-LNL, INFN-Turin, INFN-LNS, LASA at INFN-Milan, INFN-Bologna and at Elettra Sincrotrone Trieste. The authors also want to thank all colleagues at ESS in Lund and the ESS accelerator collaboration, as we have tried to describe in this paper, ESS is only made possible thanks to collaboration. The contribution of G. Torrisi to the paper revision is warmly acknowledged. The support of the INFN and Elettra management was the basis for the work here described.

Open Access This article is licensed under a Creative Commons Attribution 4.0 International License, which permits use, sharing, adaptation, distribution and reproduction in any medium or format, as long as you give appropriate credit to the original author(s) and the source, provide a link to the Creative Commons licence, and indicate if changes were made. The images or other third party material in this article are included in the article's Creative Commons licence, unless indicated otherwise in a credit line to the material. If material is not included in the article's Creative Commons licence and your intended use is not permitted by statutory regulation or exceeds the permitted use, you will need to obtain permission directly from the copyright holder. To view a copy of this licence, visit http://creativecommons.org/licenses/by/4.0/.

\section{References}

1. ESS Technical Design Report, April 23, 2013, ESS-doc-274, ISBN 978-91-980173-2-8, Executive editor: S. Peggs, Structural editor: R. Kreier

2. https://europeanspallationsource.se

3. M. Lindroos, G. Rhees, S. Molloy, M. Seidel, Elementary Particles - Accelerators and Colliders, Chapter on spallation sources, Series: Landolt-Börnstein: Numerical Data and Functional Relationships in Science and Technology - New Series, Subvolume 21C, Subseries: Elementary Particles, Nuclei and Atoms, Set: Landolt-Börnstein Set 2013 - ISBN 978-3-642-23052-3

4. L. Zanini et al, Design of the cold and thermal neutron moderators for the European Spallation Source. Nucl Inst. Methods Phys. Res. A 925, 33-52 (2019)

5. K.H. Andersen et al., Optimization of moderators and beam extraction at the ESS. J. Appl. Cryst. 51, 264-281 (2018)

6. J. Galambos, Operations experience of SNS at $1.4 \mathrm{MW}$ and upgrade plans for doubling the beam power", Proceedings of the 10th Int. Part. Accel. Conf. (IPAC), 2019, Melbourne, 4380-4384

7. M. Futakawa, F. Maekawa, S. Sakamoto, 1-MW Pulsed Spallation Neutron Source (JSNS) at J- PARC. Neutron News 22(1), 15-19 (2011)

8. K.H. Andersen, The instrument suite of the European Spallation Source. Nucl. Inst. Methods Phys. Res. A 957, 163402 (2020)

9. M. Eshraqi, Cost optimized design of high power linacs. Proc. of Int. Conf. of Linear Accelerator (LINAC), Geneva, Switzerland, pp 785-789 (2014)

10. Aurélien Ponton, Warm to cold linac transition energy: can we find an optimum? Notes of the SLHiPP Collaboration meeting, Catania, Italy, unpublished (2012)

11. R. Andersson, E. Bargalló, S. Kövecses, A. Nordt, M. Zaera-Sanz, C. Hilbes, M. Rejzek, Development and status of protection functions for the Normal Conducting Linac at ESS, Proceedings of the 8th Int. Part. Accel. Conf. (IPAC), 2017, Copenhagen, pp. 1880-1883.

12. The TRASCO_AC Group, Status of the High Current Proton Accelerator for the TRASCO Program, INFN Report INFN/TC-00/23, December 2000, unpublished 
13. J. M. Lagniel, S. Joly, J. Lemaire, A. C. Mueller, IPHI, the Saclay high-intensity proton injector project, Proc. of the Part. Acc. Conf. Vancouver, Canada, pp. 1120-1122 (1997)

14. A. Fabris et al., "ESS related Activities at Elettra Sincrotrone Trieste", Proc. of the 10th Int. Part. Accel. Conf. (IPAC), Melbourne, Australia pp. 4345-4348 (2019)

15. D. Einfeld et al., Layout of the ANKA RF System, Proc. of the 6th European Particle Accelerator Conference (EPAC), Stockholm, Sweden, pp. 1820-1822 (1998)

16. C. Pasotti et al., The RF Cavity for the SESAME Facility, Proceedings of the 8th Int. Part. Accel. Conf. (IPAC), Copenhagen, Denmark, pp. 4158-4161 (2017)

17. M. Eshraqi, R. Miyamoto, E. Sargsyan, R. De Prisco, H. D. Thomsen, Statistical error studies in the ESS Linac, Proceedings of the 5th Int. Part. Accel. Conf. (IPAC), Dresden, Germany, pp. 3323-3325 (2014)

18. Y. I. Levinsen, M. Eshraqi, R. Miyamoto, M. Munoz, A. Ponton, R. De Prisco, Proceedings of the Workshop on High Brightness Beams, Malmö, pp. 315-318 (2016)

19. M. Eshraqi et al., The ESS Linac, Proceedings of the 5th Int. Part. Accel. Conf. (IPAC), 2014, Dresden, Germany, 3320-3322

20. L. Celona et al., AIP Conf. Proc. 2011, 020019 (2018)

21. R. Miracoli et al., Characterization of Microwave Discharge Ion Source for high proton beam production. Eur. Phys. J. Plus 127, 136 (2012)

22. L. Celona, Microwave Discharge Ion Sources, Proc. of the CERN Accelerator School: Ion Sources, Senec, Slovakia, pp. 421-441 (2013)

23. R. Miyamoto et al., First results of beam commissioning on the ESS site for the ion source and low energy beam transport, Proc. 10th Int. Part. Accel. Conf., Melbourne, 1118-1121 (2019)

24. M.Eshraqi, Jour. of Surface Investigation, vol.14 suppl. 1, S42-49

25. O. Piquet et al., ESS RFQ: construction status and power couplers qualification, Proceedings of the 10th Int. Part. Accel. Conf. (IPAC), Melbourne, Australia, pp 855-857 (2019)

26. I. Bustinduy, M. Magan, F. Sordo, R. Miyamoto,"Current Status on ESS Medium Energy Beam Transport", 54th ICFA Advanced Beam Dynamics Workshop on High-Intensity and High-Brightness Hadron Beams, vol. 3, pp. 170-174 (2014)

27. F. Grespan, M. Comunian, A. Pisent, M. Poggi, C. R. Roncolato, P. Mereu, "ESS DTL Design and Drift Tube Prototypes", in Proc. 27th Linear Accelerator Conf. (LINAC'14), Geneva, Switzerland, Aug-Sep. 2014, paper THPP087, pp. 1050-1052

28. P. Duthil et al., Design and prototyping of the spoke cryomodule for ESS, Proceedings of the workshop on High Brightness Beam, Malmo, pp. 416-421 (2016)

29. P. Michelato et al., Status of the ESS medium beta cavities at INFN-LASA, Proc. of the 10th Int. Part. Accel. Conf. (IPAC), Melbourne, Australia, pp. 2211-2214 (2019)

30. C. Darve et al., ESS superconducting RF collaboration, Proc. of the 8th Int. Part. Accel. Conf. (IPAC), Copenhagen, pp. 1068-1070 (2017)

31. L Neri et al., J. Phys. Conf. Ser. 874, 012037 (2017)

32. P. Mereu et al., Proc. of Int. Conf. of Linear Accelerator (LINAC), Beijing, China, pp. 260-263 (2018)

33. G. S. Mauro et al., Field uniformity preservation strategies for the ESS DTL: approach and simulations, Proc. of the 8th Int. Part. Accel. Conf. (IPAC), Copenhagen, pp. 4139-4141 (2017)

34. P. Mereu et al., Proc. of the 7th Int. Part. Accel. Conf. (IPAC), Busan, South Korea, pp. 2131-2133 (2016)

35. C. Pasotti et al., Radio Frequency Power Stations for ESS LINAC Spoke Section, Proc. of the 10th Int. Part. Accel. Conf. (IPAC), Melbourne, Australia, pp. 4345-4348 (2019)

36. R. Yogi et al., Tetrode Based Technology Demonstrator at $352 \mathrm{MHz} 400 \mathrm{kWp}$ for ESS Spoke Linac, IEEE International Vacuum Electronic Conference (IVEC), pp. 113-115 (2014)

37. C. Pasotti, private communication

38. C.A. Martins, High Voltage Modulators ESS Developments, Talk given at ESS (Lund, Sweden, 2015). (unpublished)

39. S. Grulja et al, Wire Scanner Diagnostic System, Proc. of the 8th Int. Beam Instr. Conf. (IBIC), Malmo, Sweden, pp. 326-327 (2019)

40. D. Castronovo et al. , ESS Magnets at Elettra Sincrotrone Trieste, Proc. of the 10th Int. Part. Accel. Conf. (IPAC), Melbourne, Australia, pp. 4148-4151 (2019)

41. R. Visintini et al. , Power Converters for the ESS Warm Magnets: Procurement Status, Proc. of the 10th Int. Part. Accel. Conf. (IPAC), Melbourne, Australia, pp. 4148-4151 (2019) 
42. The Importance of Physics to the Economies of Europe, Report by CEBR (Centre for Economics and Business Research) (2019), Ed. European Physical Society, available at https://cdn.ymaws.com/www. eps.org/resource/resmgr/policy/eps_pp_physics_ecov5_full.pdf

43. M. Mazzucato, The Value of Everything: Makers and Takers in the Global Economy, (2018) Allen Lane - ISBN 978-0-241-34779-9 